\title{
Approximate Reversal of Quantum Gaussian Dynamics
}

\author{
Ludovico Lami*1, Siddhartha Das ${ }^{\dagger 2}$, and Mark M. Wilde ${ }^{\ddagger 3}$
}

1 Física Teòrica: Informació i Fenòmens Quàntics, Departament de Física, Universitat Autònoma de Barcelona, Barcelona, Spain ludovico.lami@gmail.com

2 Hearne Institute for Theoretical Physics, Department of Physics and Astronomy, Louisiana State University, Baton Rouge, USA sdas21@lsu.edu

3 Hearne Institute for Theoretical Physics, Department of Physics and Astronomy, and Center for Computation \& Technology, Louisiana State University, Baton Rouge, USA

mwilde@lsu.edu

\begin{abstract}
Recently, there has been focus on determining the conditions under which the data processing inequality for quantum relative entropy is satisfied with approximate equality. The solution of the exact equality case is due to Petz, who showed that the quantum relative entropy between two quantum states stays the same after the action of a quantum channel if and only if there is a reversal channel that recovers the original states after the channel acts. Furthermore, this reversal channel can be constructed explicitly and is now called the Petz recovery map. Recent developments have shown that a variation of the Petz recovery map works well for recovery in the case of approximate equality of the data processing inequality. Our main contribution here is a proof that bosonic Gaussian states and channels possess a particular closure property, namely, that the Petz recovery map associated to a bosonic Gaussian state $\sigma$ and a bosonic Gaussian channel $\mathcal{N}$ is itself a bosonic Gaussian channel. We furthermore give an explicit construction of the Petz recovery map in this case, in terms of the mean vector and covariance matrix of the state $\sigma$ and the Gaussian specification of the channel $\mathcal{N}$.
\end{abstract}

1998 ACM Subject Classification H.1.1 Systems and Information Theory

Keywords and phrases Gaussian dynamics, Petz recovery map

Digital Object Identifier 10.4230/LIPIcs.TQC.2017.10

\section{Introduction}

\subsection{Introduction to recoverability in quantum information}

Strong subadditivity of quantum entropy is one of the cornerstones of quantum information theory, on which many fundamental results rely. Defining the conditional mutual information of a tripartite state $\rho_{A B C}$ as

$$
I(A ; B \mid C)_{\rho}:=S(A C)_{\rho}+S(B C)_{\rho}-S(A B C)_{\rho}-S(C)_{\rho},
$$

\footnotetext{
* LL acknowledges financial support from the European Research Council (AdG IRQUAT No. 267386), the Spanish MINECO (Project no. FIS2013-40627-P and no. FIS2016-86681-P), and the Generalitat de Catalunya (CIRIT Project no. 2014 SGR 966).

$\dagger$ SD acknowledges support from the Economic Development Assistantship of Louisiana State University. $¥$ MMW acknowledges support from the National Science Foundation under Award No. CCF-1350397.

(c) (i) $\odot$ Ludovico Lami, Siddhartha Das, and Mark M. Wilde;

12th Conference on the Theory of Quantum Computation, Communication, and Cryptography (TQC 2017). Editor: Mark M. Wilde; Article No. 10; pp. 10:1-10:18

Leibniz International Proceedings in Informatics

LI I ICS Schloss Dagstuhl - Leibniz-Zentrum für Informatik, Dagstuhl Publishing, Germany
} 
where $S(G)_{\sigma} \equiv-\operatorname{Tr}\left[\sigma_{G} \log \sigma_{G}\right]$ is the quantum entropy of a state $\sigma_{G}$ of a system $G$, strong subadditivity is equivalent to the non-negativity of conditional mutual information: $I(A ; B \mid C)_{\rho} \geq 0$. Initially conjectured in $1967[55,26]$, it was subsequently proven six years later $[35,36]$. Afterward, its equivalence to the data processing inequality for the quantum relative entropy [68] was realized $[66,37,38,56]$. This latter inequality has the form

$$
D(\rho \| \sigma) \geq D(\mathcal{N}(\rho) \| \mathcal{N}(\sigma))
$$

being valid for all states $\rho, \sigma$ and all quantum channels $\mathcal{N}$ (completely positive, tracepreserving maps). Here, the quantum relative entropy is defined for quantum states $\rho$ and $\sigma$ as

$$
D(\rho \| \sigma) \equiv \operatorname{Tr}[\rho(\log \rho-\log \sigma)],
$$

whenever the support of $\rho$ is contained in the support of $\sigma$, and it is set to $+\infty$ otherwise [68].

The interest in strong subadditivity has not fallen over time, and many different proofs for it have been proposed in the last four decades (see for instance [43]). At the same time, new improvements of the original inequality have recently been found. Extending methods originally proposed in [17], an operator generalization of strong subadditivity was recently proven in [28].

A line of research which is of particular interest to us focuses on investigating the conditions under which strong subadditivity, or more generally the data processing inequality for relative entropy, is satisfied with equality or approximate equality. The solution of the exact equality case dates back to the 1980s: in [50,51,52], it was shown that the relative entropy between two states stays the same after the action of a quantum channel if and only if there is a recovery channel bringing back both images to the original states. Furthermore, this reversing channel can be constructed explicitly and now takes the name Petz recovery map. Afterward, $[42,41]$ proved a structure theorem giving a form for states and a channel saturating the data-processing inequality for relative entropy, and, related to this development, the form of tripartite states satisfying strong subadditivity with equality was determined in [24].

Characterising the structure of states for which strong subadditivity is nearly saturated requires different techniques, and progress was not made until more recently. In 2011, a lower bound on conditional mutual information in terms of one-way LOCC norms [40] was proven in [9], the motivation for [9] lying in the question of faithfulness of an entanglement measure called squashed entanglement [14] (see also [64, 65] for discussions related to squashed entanglement). Later on, a conjecture put forward in [75] proposed another operationally meaningful remainder term for the relative entropy decrease induced by a quantum channel, given by the relative entropy between the state $\rho$ and a "recovered version" of $\mathcal{N}(\rho)$. The authors of [75] proposed the following conjecture as a refinement of (2):

$$
D(\rho \| \sigma) \stackrel{?}{\geq} D(\mathcal{N}(\rho) \| \mathcal{N}(\sigma))+D\left(\rho \|\left(\mathcal{R}_{\sigma, \mathcal{N}} \circ \mathcal{N}\right)(\rho)\right),
$$

where $\mathcal{R}_{\sigma, \mathcal{N}}$ should be a quantum channel depending only on $\sigma$ and $\mathcal{N}$ and such that $\left(\mathcal{R}_{\sigma, \mathcal{N}} \circ \mathcal{N}\right)(\sigma)=\sigma$. The authors of [75] proved (4) in the classical case, when the states $\rho$ and $\sigma$ commute and the channel is classical as well, and they showed how the recovery channel in this case can be taken as the Petz recovery map. This conjecture has now been proven in a number of special, yet physically relevant cases as well $[2,11,39,32,3]$. Unfortunately, the authors of [75] showed that in the general quantum case, $\mathcal{R}_{\sigma, \mathcal{N}}$ in (4) cannot be taken as the Petz recovery map. For further details, see also [29, 33], and for related conjectures, see $[7,59]$. 
While the general form of the conjecture in (4) remains unproven, in [18], it was shown that if the conditional mutual information $I(A ; B \mid C)_{\rho}$ is small, then the state $\rho_{A B C}$ can be very well approximated by one of its "reconstructed" versions $\mathcal{R}_{C \rightarrow B C}\left(\rho_{A C}\right)$. That is, the authors of [18] proved the following inequality:

$$
I(A ; B \mid C)_{\rho} \geq-\log F\left(\rho_{A B C}, \mathcal{R}_{C \rightarrow B C}\left(\rho_{A C}\right)\right),
$$

where $F$ denotes the quantum fidelity [67], defined as $F(\omega, \tau):=\|\sqrt{\omega} \sqrt{\tau}\|_{1}^{2}$ for quantum states $\omega$ and $\tau$, and $\mathcal{R}_{C \rightarrow B C}$ is a recovery channel taking an input system $C$ to output systems $B C$. Furthermore, the channel $\mathcal{R}_{C \rightarrow B C}$ can be taken as the Petz recovery map up to some unitary rotations preceding and following its action, but note that the unitary rotations given in [18] generally depend on the full state $\rho_{A B C}$.

After the result of [18] appeared, much activity surrounding entropy inequalities and recovery channels occurred. An alternative and simpler proof of the faithfulness of squashed entanglement following the lines of [75] immediately appeared [33], while an alternative proof of (5) that makes use of quantum state redistribution [15, 76] appeared in [10]. In [62], an important particular case of (5) was proven; that is, it was shown that the recovery map in (5) can be chosen to depend only on $\rho_{B C}$ and to obey $\mathcal{R}_{C \rightarrow B C}\left(\rho_{C}\right)=\rho_{B C}$. A different approach was delivered in [71], based on the methods of complex interpolation [6] and generalized Rényi entropies [7, 59]. The main result of [71] states that a lower bound on the decrease in relative entropy induced by a quantum channel is given by the negative logarithm of the fidelity between the first state and its recovered version, which is a step closer to the proof of the conjecture in (4). However, the recovery term in [71] is weaker than the right-hand side of (4), and the map appearing in it lacks one of the two properties that it is required to obey. Another step toward the proof of the conjecture in (4) was performed in [27], where a more general tool from complex analysis [25] and the methods of [7, 59, 71] were exploited in order to prove a statement similar to (4), with the relative entropy on the right-hand side substituted by a negative log-fidelity, but with the recovery map depending only on $\sigma$ and $\mathcal{N}$ and furthermore satisfying $\mathcal{R}_{\sigma, \mathcal{N}}(\mathcal{N}(\sigma))=\sigma$. Meanwhile, a different proof approach based on pinching was delivered in [63], and then a systematic method for deriving matrix inequalities by forcing the operators to commute via the application of suitably chosen "pinching maps" was proposed in [61]. This method as well as the complex interpolation techniques in [16] can be also applied to prove multioperator trace inequalities $[16,61,72]$, which generalise the celebrated Golden-Thompson inequality $\operatorname{Tr}\left[e^{X+Y}\right] \leq \operatorname{Tr}\left[e^{X} e^{Y}\right](X, Y$ hermitian) and the stronger statements given in [34]. The results of [61] also marked further progress toward establishing the conjecture in (4).

\subsection{Introduction to quantum Gaussian states and channels}

A major platform for the application of quantum information theory to physical information processing is constituted by quantum optics [20] with a finite number of electromagnetic modes or quantum harmonic oscillators. From the mathematical perspective, this framework can be thought of as quantum mechanics applied to separable Hilbert spaces endowed with a finite number of operators obeying canonical commutation relations [58].

A typical free Hamiltonian of such a system is quadratic in the canonical operators, and in fact, a special role within this context is played by ground or thermal states of such Hamiltonians, commonly called Gaussian states. These states define a useful operational framework for several reasons, stemming from both physics and mathematics $[1,58]$. From the physical point of view, they are easily produced and manipulated in the laboratory and 
can be used to implement effective quantum protocols [4, 69]. Mathematically convenient properties that qualify them as defining a legitimate framework include

1. the closure under so-called Gaussian unitary evolutions, that is, unitaries induced by piecewise time evolution via quadratic Hamiltonians, as well as more generally

2. the closure under Gaussian channels, which can be understood as the operation of adding an ancillary system in a vacuum state, applying a global Gaussian unitary, and tracing out one of the subsystems [12].

Recently, more advanced "closure" properties have been established, such as the optimality of Gaussian states for optimising the output entropy of one-mode, phase-covariant quantum channels, even when a fixed value of the input entropy is prescribed [23, 48, 46, 47]. These facts have the striking implication that it suffices to select coding strategies according to Gaussian states in order to achieve optimal rates in several quantum communication tasks $[22,73,21,54,74,47]$.

\subsection{Summary of main result}

The main contribution of our paper is a proof that Gaussian states and channels possess another closure property: the Petz recovery map associated to a Gaussian state $\sigma$ and a Gaussian channel $\mathcal{N}$ is itself a Gaussian channel (see Theorem 1). Additionally, we achieve this result through an explicit construction of the action of such a Gaussian Petz channel, which lends itself to multiple applications. For instance, with the formulas we provide, it is possible to construct a counterexample to the inequality in (4), in which all the states and channels involved are Gaussian and $\mathcal{R}_{\sigma, \mathcal{N}}$ is the Petz recovery map. This is similar to what happens in the finite-dimensional case. Another application of our main result is a more explicit form for an entropy inequality from [27], whenever the states and channel involved are Gaussian.

This paper is structured as follows. In Section 2, we review some background material and establish notation. In particular, we review the Petz recovery map (Section 2.1) and bosonic Gaussian states and channels (Section 2.2). In Section 3, we state our main result, Theorem 1, which establishes that the Petz recovery map for a Gaussian state $\sigma$ and a Gaussian channel $\mathcal{N}$ is itself a Gaussian channel, and we give an explicit form for it in terms of the parameters that characterize $\sigma$ and $\mathcal{N}$. Corollary 2 establishes a similar result for the rotated Petz maps from [71]. For our detailed proof of Theorem 1, we refer to [30, Sections 3.1-3.4]. We conclude in Section 4 with a summary and some open questions.

\section{Background and notation}

\subsection{Petz recovery map}

As discussed in Section 1.1, the Petz recovery map is a notable object playing a crucial role in the theory of quantum recoverability. It has been interpreted in [31] as a quantum generalization of the Bayes rule from probability theory. Given a state $\sigma$ and a channel $\mathcal{N}$, the associated Petz map $\mathcal{P}_{\sigma, \mathcal{N}}$ is defined as a linear map satisfying the following [50, 51, 44]:

$$
\left\langle A, \mathcal{N}^{\dagger}(B)\right\rangle_{\sigma}=\left\langle\mathcal{P}_{\sigma, \mathcal{N}}^{\dagger}(A), B\right\rangle_{\mathcal{N}(\sigma)}, \quad \forall A, B,
$$

where $A$ and $B$ are bounded operators and the weighted Hilbert-Schmidt inner product is defined for bounded operators $\tau_{1}$ and $\tau_{2}$ and a trace-class operator $\xi$ as

$$
\left\langle\tau_{1}, \tau_{2}\right\rangle_{\xi} \equiv \operatorname{Tr}\left[\tau_{1}^{\dagger} \xi^{1 / 2} \tau_{2} \xi^{1 / 2}\right] .
$$


The map $\mathcal{P}_{\sigma, \mathcal{N}}$ is unique if $\mathcal{N}(\sigma)$ is a faithful operator $[50,51,44]$, and otherwise, it is unique on the support of this operator. If $\sigma$ acts on a finite-dimensional Hilbert space and $\mathcal{N}$ is a quantum channel with finite-dimensional inputs and outputs, then the Petz map takes the following explicit form [24]:

$$
\mathcal{P}_{\sigma, \mathcal{N}}(\omega) \equiv \sigma^{1 / 2} \mathcal{N}^{\dagger}\left(\mathcal{N}(\sigma)^{-1 / 2} \omega \mathcal{N}(\sigma)^{-1 / 2}\right) \sigma^{1 / 2}
$$

where $\mathcal{N}(\sigma)^{-1 / 2}$ is understood as a generalized inverse (i.e., inverse on the support of $\mathcal{N}(\sigma)$ ). Sometimes we the dependence of $\mathcal{P}$ on $\sigma$ and $\mathcal{N}$ for the sake of simplicity. A rotated Petz $\operatorname{map} \mathcal{P}_{\sigma, \mathcal{N}}^{t}$ for $t \in \mathbb{R}$, a state $\sigma$, and a channel $\mathcal{N}$ is defined as [71]

$$
\mathcal{P}_{\sigma, \mathcal{N}}^{t}(\omega) \equiv \sigma^{i t} \mathcal{P}_{\sigma, \mathcal{N}}\left(\mathcal{N}(\sigma)^{-i t} \omega \mathcal{N}(\sigma)^{i t}\right) \sigma^{-i t},
$$

with $\sigma^{i t}=\exp (i t \log \sigma)$ being understood as a unitary evolution according to the Hamiltonian $\log \sigma$.

\subsection{Quantum Gaussian states and channels}

Here we provide some background on quantum Gaussian states and channels (see [12, 1, 58] for reviews). An $n$-mode quantum system is described by a density operator acting on a tensorproduct Hilbert space. To the $j$ th Hilbert space in the tensor product, for $j \in\{1, \ldots, n\}$, we let $x_{j}$ and $p_{j}$ denote the position- and momentum-quadrature operator, respectively. These operators satisfy the canonical commutation relations: $\left[x_{j}, p_{k}\right]=i \delta_{j, k}$, where we have set $\hbar=1$. It is convenient to form a vector $r=\left(x_{1}, \ldots, x_{n}, p_{1}, \ldots, p_{n}\right)^{T}$ from these operators, and then we can rewrite the canonical commutation relations in matrix form as follows:

$$
\left[r, r^{T}\right]=i \Omega
$$

where

$$
\Omega \equiv\left[\begin{array}{cc}
0 & 1 \\
-1 & 0
\end{array}\right] \otimes I_{n},
$$

and $I_{n}$ denotes the $n \times n$ identity matrix. We often make use of the identities $\Omega^{T} \Omega=I$ and $\Omega^{T}=-\Omega$.

The displacement (Weyl) operator $D_{z}$ plays an important role in Gaussian quantum information, defined for $z \in \mathbb{R}^{2 n}$ as

$$
D_{z} \equiv \exp \left(i z^{T} \Omega r\right)
$$

For $z_{1}, z_{2} \in \mathbb{R}^{2 n}$, the displacement operators satisfy the following composition rule:

$$
D_{z_{1}} D_{z_{2}}=D_{z_{1}+z_{2}} e^{-\frac{i}{2} z_{1}^{T} \Omega z_{2}} .
$$

It can be shown that displacement operators form a complete, orthogonal set of operators, and their Hilbert-Schmidt orthogonality relation is as follows:

$$
\operatorname{Tr}\left[D_{z_{1}} D_{-z_{2}}\right]=(2 \pi)^{n} \delta\left(z_{1}-z_{2}\right) .
$$

Moreover, due to their completeness, these operators allow for a Fourier-Weyl expansion of a quantum state, in terms of a characteristic function. In more detail, a quantum state $\rho$ has a characteristic function $\chi_{\rho}(w)$, defined as

$$
\chi_{\rho}(w) \equiv \operatorname{Tr}\left[\rho D_{-w}\right]
$$


and the original state $\rho$ can be written in terms of $\chi_{\rho}(w)$ as

$$
\rho=\int \frac{d^{2 n} w}{(2 \pi)^{n}} \chi_{\rho}(w) D_{w} .
$$

The mean vector $s_{\rho} \in \mathbb{R}^{2 n}$ and $2 n \times 2 n$ covariance matrix $V_{\rho}$ of a quantum state $\rho$ are defined as

$$
\begin{aligned}
s_{\rho} & \equiv\langle r\rangle_{\rho}=\operatorname{Tr}[r \rho] \\
V_{\rho} & \equiv\left\langle\left\{r-s_{\rho}, r^{T}-s_{\rho}^{T}\right\}\right\rangle_{\rho}=\operatorname{Tr}\left[\left\{r-s_{\rho}, r^{T}-s_{\rho}^{T}\right\} \rho\right] .
\end{aligned}
$$

It follows from the above definition that the covariance matrix $V_{\rho}$ is symmetric.

A quantum Gaussian state is a ground or thermal state of a Hamiltonian that is quadratic in the position- and momentum-quadrature operators. In particular, up to an irrelevant additive constant, any such Hamiltonian has the form $\frac{1}{2}(r-s)^{T} H(r-s)$, where $s \in \mathbb{R}^{2 n}$ and $H$ is a $2 n \times 2 n$ positive definite matrix that we refer to as the Hamiltonian matrix. Then a quantum Gaussian state $\rho$ takes the form

$$
\rho=Z_{\rho}^{-1} \exp \left(-\frac{1}{2}\left(r-s_{\rho}\right)^{T} H_{\rho}\left(r-s_{\rho}\right)\right)
$$

where $Z_{\rho} \equiv \operatorname{Tr}\left[\exp \left(-\frac{1}{2}\left(r-s_{\rho}\right)^{T} H_{\rho}\left(r-s_{\rho}\right)\right)\right]$ and one can show that $\langle r\rangle_{\rho}=s_{\rho} \in \mathbb{R}^{2 n}$ (i.e., $s_{\rho}$ is the mean vector of $\rho$ ). Defining

$$
V_{\rho} \equiv \operatorname{coth}\left(\frac{i \Omega H_{\rho}}{2}\right) i \Omega
$$

one can also show that $V_{\rho}$ is the covariance matrix of $\rho$, whose matrix elements satisfy $V_{\rho}^{j, k}=\left\langle\left\{r_{j}-s_{\rho}^{j}, r_{k}-s_{\rho}^{k}\right\}\right\rangle_{\rho}$ and the Heisenberg uncertainty relation [60]:

$$
V_{\rho}+i \Omega \geq 0
$$

A quantum Gaussian state is faithful (having full support) if $V_{\rho}+i \Omega>0$.

A quantum Gaussian state $\rho$ with mean vector $s_{\rho}$ and covariance matrix $V_{\rho}$ has the following Gaussian characteristic function:

$$
\chi_{\rho}(w)=\exp \left(-\frac{1}{4}(\Omega w)^{T} V_{\rho} \Omega w+i(\Omega w)^{T} s_{\rho}\right),
$$

so that it can be written in the following way:

$$
\rho=\int \frac{d^{2 n} w}{(2 \pi)^{n}} \exp \left(-\frac{1}{4}(\Omega w)^{T} V_{\rho} \Omega w+i(\Omega w)^{T} s_{\rho}\right) D_{w} .
$$

After a change of variables $(w \rightarrow \Omega w)$, this representation becomes

$$
\rho=\int \frac{d^{2 n} w}{(2 \pi)^{n}} \exp \left(-\frac{1}{4} w^{T} V_{\rho} w-i w^{T} s_{\rho}\right) D_{\Omega w} .
$$

A quantum Gaussian channel is a completely positive, trace-preserving map that takes Gaussian input states to Gaussian output states. A quantum Gaussian channel $\mathcal{N}$ that takes $n$-mode Gaussian input states to $m$-mode Gaussian output states is specified by a $2 m \times 2 n$ transformation matrix $X$, a $2 m \times 2 m$ positive semi-definite, additive noise matrix $Y$, and a displacement vector $\delta \in \mathbb{R}^{2 n}$. The action of such a channel on a generic state $\rho$ with 
characteristic function $\chi_{\rho}(w)$ is to output a state $\mathcal{N}(\rho)$ having the following characteristic function:

$$
\chi_{\mathcal{N}(\rho)}(w)=\chi_{\rho}\left(\Omega^{T} X^{T} \Omega w\right) \exp \left(-\frac{1}{4}(\Omega w)^{T} Y \Omega w+i(\Omega w)^{T} \delta\right) .
$$

Then the channel $\mathcal{N}$ leads to the following transformation of the covariance matrix $V$ and mean vector $s$ of an input quantum Gaussian state:

$$
\mathcal{N}:\left\{\begin{array}{lll}
V & \longmapsto X V X^{T}+Y \\
s & \longmapsto X s+\delta
\end{array}\right.
$$

The matrices $X$ and $Y$ should satisfy the following condition in order for the map $\mathcal{N}$ to be completely positive:

$$
Y+i \Omega \geq i X \Omega X^{T} .
$$

The adjoint of a quantum channel $\mathcal{N}$ is defined as the unique linear map satisfying the following for all $A$ and $B$ :

$$
\langle A, \mathcal{N}(B)\rangle=\left\langle\mathcal{N}^{\dagger}(A), B\right\rangle,
$$

where $B$ is an arbitrary trace-class operator, $A$ is an arbitrary bounded operator, and the Hilbert-Schmidt inner product is defined for operators $A_{1}$ and $A_{2}$ as $\left\langle A_{1}, A_{2}\right\rangle \equiv \operatorname{Tr}\left[A_{1}^{\dagger} A_{2}\right]$. The adjoint map $\mathcal{N}^{\dagger}$ is completely positive and unital if $\mathcal{N}$ is completely positive and tracepreserving. The action of the adjoint $\mathcal{N}^{\dagger}$ of a quantum Gaussian channel $\mathcal{N}$ defined by (26) is as follows $[12,19]$, when acting on a displacement operator $D_{\Omega z}$ :

$$
\mathcal{N}^{\dagger}\left(D_{\Omega z}\right)=D_{\Omega X^{T} z} \exp \left(-\frac{1}{4} z^{T} Y z+i z^{T} \delta\right) .
$$

The action of the adjoint $\mathcal{N}^{\dagger}$ on a quantum Gaussian state with covariance matrix $V$ and mean vector $s$ is then to output a quantum Gaussian operator described by covariance matrix $X^{-1}(V+Y) X^{-T}$ and mean vector $X^{-1}(s-\delta)$ whenever $X$ is invertible [19, Appendix B]. We summarize these transformation rules as follows:

$$
\mathcal{N}^{\dagger}:\left\{\begin{array}{lll}
V & \longmapsto & X^{-1}(V+Y) X^{-T} \\
s & \longmapsto X^{-1}(s-\delta)
\end{array} .\right.
$$

Typically one thinks of the channel $\mathcal{N}$ as acting in the Schrödinger picture, taking input states to output states, and one thinks of the adjoint $\mathcal{N}^{\dagger}$ as acting in the Heisenberg picture, taking input bounded operators to output bounded operators. So this is why we have specified the channel $\mathcal{N}$ in terms of its action on characteristic functions, which describe states, and the adjoint $\mathcal{N}^{\dagger}$ in terms of its action on displacement operators, a natural choice of bounded operators in our context here.

Often we find it useful to write

$$
\sigma=D_{s_{\sigma}}^{\dagger} \sigma_{0} D_{s_{\sigma}}
$$

where $\sigma_{0}$ is a Gaussian state with the same covariance matrix as $\sigma$ but with vanishing mean vector. Analogously, the channel $\mathcal{N}$ in (25) admits the following decomposition:

$$
\mathcal{N}(\cdot)=D_{\delta}^{\dagger} \mathcal{N}_{0}(\cdot) D_{\delta},
$$


where $\mathcal{N}_{0}$ is a zero-displacement Gaussian channel, acting as in (26) but with $\delta=0$. Taking the adjoint gives

$$
\mathcal{N}^{\dagger}(\cdot)=\mathcal{N}_{0}^{\dagger}\left(D_{\delta}(\cdot) D_{\delta}^{\dagger}\right)
$$

Applying $\mathcal{N}$ to $\sigma$ yields

$$
\mathcal{N}(\sigma)=D_{X s+\delta}^{\dagger} \mathcal{N}_{0}\left(\sigma_{0}\right) D_{X s+\delta},
$$

which follows from (26). We also make use of the following channel covariance relations:

$$
\begin{aligned}
\mathcal{N}\left(D_{\gamma}^{\dagger}(\cdot) D_{\gamma}\right) & =D_{X \gamma+\delta}^{\dagger} \mathcal{N}_{0}(\cdot) D_{X \gamma+\delta}, \\
\mathcal{N}^{\dagger}\left(D_{\gamma}^{\dagger}(\cdot) D_{\gamma}\right) & =D_{X^{-1}(\gamma-\delta)}^{\dagger} \mathcal{N}_{0}^{\dagger}(\cdot) D_{X^{-1}(\gamma-\delta)},
\end{aligned}
$$

which follow from (25), (26), (29), and (30). Note that (36) holds whenever $X$ is invertible.

Finally, given a Gaussian state $\sigma$ with mean vector $s_{\sigma}$ and covariance matrix $V_{\sigma}$, we can consider a unitary rotation of the form $\sigma^{i t}=\exp (i t \log \sigma)$ for $t \in \mathbb{R}$. By using the representation in (19) with the Hamiltonian matrix $H_{\sigma}$, we can write the unitary $\sigma^{i t}$ as

$$
\begin{aligned}
\sigma^{i t} & =\exp \left(-\frac{i}{2}\left(r-s_{\sigma}\right)^{T} H_{\sigma} t\left(r-s_{\sigma}\right)\right) \exp \left(-i t \log Z_{\sigma}\right) \\
& =D_{-s_{\sigma}}\left[\exp \left(\frac{i}{2} r^{T}\left(-H_{\sigma} t\right) r\right) \exp \left(-i t \log Z_{\sigma}\right)\right] D_{s_{\sigma}}
\end{aligned}
$$

where we have used the fact that $\left(r-s_{\sigma}\right)^{T} H_{\sigma}\left(r-s_{\sigma}\right)=D_{-s_{\sigma}} r^{T} H_{\sigma} r D_{s_{\sigma}}$ and the operator identity $B \exp (A) B^{-1}=\exp \left(B A B^{-1}\right)$. The unitary $\sigma^{i t}$ is a Gaussian unitary because it is generated by a Hamiltonian no more than quadratic in the position- and momentumquadrature operators. Let us define the symplectic transformation corresponding to the unitary $\exp \left(\frac{i}{2} r^{T}\left(-H_{\sigma} t\right) r\right)$ as

$$
S_{\sigma, t} \equiv \exp \left(\Omega H_{\sigma} t\right)
$$

so that

$$
\sigma^{i t} r \sigma^{-i t}=S_{\sigma,-t}\left(r-s_{\sigma}\right)+s_{\sigma},
$$

where we used that $D_{s_{\sigma}} r D_{-s_{\sigma}}=r+s_{\sigma}$. The above formula implies that

$$
\begin{aligned}
V_{\sigma^{i t} \omega \sigma^{-i t}} & =S_{\sigma, t} V_{\omega} S_{\sigma, t}^{T}, \\
s_{\sigma^{i t} \omega \sigma^{-i t}} & =S_{\sigma, t}\left(s_{\rho}-s_{\sigma}\right)+s_{\sigma} .
\end{aligned}
$$

\section{Main result: Petz map as a quantum Gaussian channel}

Our main result is the following theorem:

- Theorem 1. Let $\sigma$ be a quantum Gaussian state with mean vector $s_{\sigma}$ and covariance matrix $V_{\sigma}$, and let $\mathcal{N}$ be a quantum Gaussian channel with its action on an input state as described in (26). Suppose furthermore that $\mathcal{N}(\sigma)$ is a faithful quantum state. Then the Petz recovery map $\mathcal{P}_{\sigma, \mathcal{N}}$ is a quantum Gaussian channel with the following action:

$$
\mathcal{P}_{\sigma, \mathcal{N}}:\left\{\begin{array}{rll}
V & \longmapsto X_{P} V X_{P}^{T}+Y_{P} \\
s & \longmapsto X_{P} s+\delta_{P}
\end{array}\right.
$$


where

$$
\begin{aligned}
X_{P} & \equiv \sqrt{I+\left(V_{\sigma} \Omega\right)^{-2}} V_{\sigma} X^{T}{\sqrt{I+\left(\Omega V_{\mathcal{N}(\sigma)}\right)^{-2}}}^{-1} V_{\mathcal{N}(\sigma)}^{-1}, \\
Y_{P} & \equiv V_{\sigma}-X_{P} V_{\mathcal{N}(\sigma)} X_{P}^{T} \\
\delta_{P} & \equiv s_{\sigma}-X_{P}\left(X s_{\sigma}+\delta\right) \\
V_{\mathcal{N}(\sigma)} & =X V_{\sigma} X^{T}+Y .
\end{aligned}
$$

That is, $\mathcal{P}_{\sigma, \mathcal{N}}$ in (43) is the unique linear map satisfying (6) for $\sigma$ and $\mathcal{N}$ as described above.

The following corollary is a direct consequence of Theorem 1 and the discussion surrounding (37)-(40):

- Corollary 2. For $\sigma$ and $\mathcal{N}$ as given in Theorem 1, the rotated Petz map $\mathcal{P}_{\sigma, \mathcal{N}}^{t}$ (defined in (9)) is also a quantum Gaussian channel with the same action as the Petz recovery channel $\mathcal{P}_{\sigma, \mathcal{N}}$ but with the substitutions

$$
\begin{aligned}
X_{P} & \rightarrow X_{P}^{t} \equiv S_{\sigma, t} X_{P} S_{\mathcal{N}(\sigma),-t}, \\
Y_{P} & \rightarrow Y_{P}^{t} \equiv S_{\sigma, t} Y_{P} S_{\sigma, t}^{T} \\
\delta_{P} & \rightarrow \delta_{P}^{t} \equiv s_{\sigma}-X_{P}^{t}\left(X s_{\sigma}+\delta\right) .
\end{aligned}
$$

That is, $\mathcal{P}_{\sigma, \mathcal{N}}^{t}$ is a quantum Gaussian channel with the following action:

$$
\mathcal{P}_{\sigma, \mathcal{N}}^{t}:\left\{\begin{array}{rl}
V & \longmapsto X_{P}^{t} V\left(X_{P}^{t}\right)^{T}+Y_{P}^{t} \\
s & \longmapsto X_{P}^{t} s+\delta_{P}^{t}
\end{array} .\right.
$$

Remark. The following entropy inequality was proven to hold whenever $\rho$ and $\sigma$ are density operators and $\mathcal{N}$ is a quantum channel [27]:

$$
D(\rho \| \sigma) \geq D(\mathcal{N}(\rho) \| \mathcal{N}(\sigma))-\int_{\mathbb{R}} d t p(t) \log F\left(\rho,\left(\mathcal{P}_{\sigma, \mathcal{N}}^{t / 2} \circ \mathcal{N}\right)(\rho)\right),
$$

where $p(t):=\frac{\pi}{2}(\cosh (\pi t)+1)^{-1}$ is a probability distribution parametrized by $t \in \mathbb{R}$. In the case that $\rho$ and $\sigma$ are quantum Gaussian states and $\mathcal{N}$ is a quantum Gaussian channel, Corollary 2 allows us to conclude that $\mathcal{P}_{\sigma, \mathcal{N}}^{t / 2}$ is a quantum Gaussian channel for all $t \in \mathbb{R}$. Furthermore, there are explicit, compact formulas for the relative entropy [57, 13, 53] and fidelity $[49,70,5]$ of two quantum Gaussian states. In both cases, the formulas are given exclusively in terms of the mean vectors and covariance matrices of the involved states. Thus, when the states and channel involved are all Gaussian, the above inequality can be rewritten in a simpler form involving only finite-dimensional matrices instead of trace-class operators acting on infinite-dimensional Hilbert spaces.

The forthcoming subsections sketch the first steps of our proof of Theorem 1, and a detailed, complete proof can be found in [30]. Before delving into our proof, we highlight our proof strategy, which proceeds according to the following steps:

1. Even though the explicit form of the Petz map in (8) is not generally valid in the infinitedimensional case because the inverse of a density operator may be unbounded, we work with it anyway, as an ansatz (call this Ansatz 1). Under Ansatz 1, we first show that it suffices to consider the case when the state $\sigma$ is a zero-mean Gaussian state and the channel $\mathcal{N}$ does not apply any displacement to the mean vector of its input, so that $s_{\sigma}=0$ and $\delta=0$, with $\delta$ defined in (25) and (26). 
2. Under the same Ansatz 1, we arrive at the hypothesis that (43) gives the explicit form for the action of the Petz map on a Gaussian input state. Recall from (8) that the Petz map is a serial concatenation of three completely positive maps:

$$
\begin{aligned}
& (\cdot) \rightarrow \mathcal{N}(\sigma)^{-1 / 2}(\cdot) \mathcal{N}(\sigma)^{-1 / 2} \\
& (\cdot) \rightarrow \mathcal{N}^{\dagger}(\cdot) \\
& (\cdot) \rightarrow \sigma^{1 / 2}(\cdot) \sigma^{1 / 2} .
\end{aligned}
$$

To handle the first completely positive map in (53), we proceed with an additional ansatz (Ansatz 2) that taking the inverse of a Gaussian state corresponds to negating its covariance matrix. This is motivated by the representation in (19), in which inverting the density operator has the effect of negating the Hamiltonian matrix, which in turn has the effect of negating the covariance matrix due to the fact that arcoth is an odd function. Furthermore, results of [5, Appendix B-2] allow us to conclude that sandwiching a Gaussian state by the square root of another Gaussian state is a Gaussian map resulting in another unnormalized, Gaussian state. To handle the second map in (54), we can directly apply a result given in [19, Appendix B], which gives an explicit form for the action of the adjoint of a Gaussian channel on a Gaussian state (see also the review in (30)). We also work with a final Ansatz 3, which is the assumption that the matrix X in (26) is invertible. Later, we show how this assumption is not necessary. To handle the third completely positive map in (55), we again apply the aforementioned result about sandwiching a Gaussian state by the square root of another.

3. After arriving at an explicit form for the Petz map by using Ansatzes 1-3, we verify that this explicit form satisfies the equations in (6) whenever the operators $A$ and $B$ are Hilbert-Schmidt operators.

4. We finally employ a limiting argument to conclude that if (6) is satisfied when $A$ and $B$ are Hilbert-Schmidt operators, then the equations are satisfied when $A$ and $B$ are arbitrary bounded operators. By a result of $[50,51,44]$, we can finally conclude that the Gaussian channel given in Theorem 1 is the unique quantum channel satisfying (6). This step then concludes our proof of Theorem 1.

In the subsections that follow, we provide details of the first two steps above, and we refer to [30] for the rest of the steps of our proof of Theorem 1.

\subsection{Step 1: Sufficiency of focusing on zero-mean Gaussian states and zero-displacement Gaussian channels}

As mentioned above, we employ Ansatz 1 in this first step, in which we work with the explicit form of the Petz map in (8), in spite of the fact that the inverse of a Gaussian density operator is unbounded. Let $\sigma$ be a quantum Gaussian state with mean vector $s_{\sigma}$ and covariance matrix $V_{\sigma}$, and let $\mathcal{N}$ be a quantum Gaussian channel with the action on an input state as described in (26).

In this first step, we show how it suffices to consider the case $s_{\sigma}=\delta=0$ in (8). To see this, consider the action of the Petz map $\mathcal{P}_{\sigma, \mathcal{N}}$ on an arbitrary input state $\omega$ : 


$$
\begin{aligned}
\mathcal{P}_{\sigma, \mathcal{N}}(\omega)= & \sigma^{1 / 2} \mathcal{N}^{\dagger}\left(\mathcal{N}(\sigma)^{-1 / 2} \omega \mathcal{N}(\sigma)^{-1 / 2}\right) \sigma^{1 / 2} \\
= & \left(D_{s_{\sigma}}^{\dagger} \sigma_{0}^{1 / 2} D_{s_{\sigma}}\right) \mathcal{N}_{0}^{\dagger}\left[D_{\delta} D_{X s_{\sigma}+\delta}^{\dagger} \mathcal{N}_{0}\left(\sigma_{0}\right)^{-1 / 2} D_{X s_{\sigma}+\delta} \omega D_{X s_{\sigma}+\delta}^{\dagger} \mathcal{N}_{0}\left(\sigma_{0}\right)^{-1 / 2} D_{X s_{\sigma}+\delta} D_{\delta}^{\dagger}\right] \\
& \quad \times\left(D_{s_{\sigma}}^{\dagger} \sigma_{0}^{1 / 2} D_{s_{\sigma}}\right) \\
= & \left(D_{s_{\sigma}}^{\dagger} \sigma_{0}^{1 / 2} D_{s_{\sigma}}\right) \mathcal{N}_{0}^{\dagger}\left[D_{X s_{\sigma}}^{\dagger} \mathcal{N}_{0}\left(\sigma_{0}\right)^{-1 / 2} D_{X s_{\sigma}+\delta} \omega D_{X s_{\sigma}+\delta}^{\dagger} \mathcal{N}_{0}\left(\sigma_{0}\right)^{-1 / 2} D_{X s_{\sigma}}\right] \\
& \quad \times\left(D_{s_{\sigma}}^{\dagger} \sigma_{0}^{1 / 2} D_{s_{\sigma}}\right) \\
= & D_{s_{\sigma}}^{\dagger} \sigma_{0}^{1 / 2} D_{s_{\sigma}} D_{X-1}^{\dagger}{ }^{-1}\left(X s_{\sigma}\right) \\
& \quad \times D_{X}^{-1}\left(X s_{\sigma}\right) \\
= & \left.D_{s_{\sigma}}^{\dagger} \sigma_{0}^{1 / 2} D_{s_{\sigma}}^{\dagger} \mathcal{N}_{0}\left(\sigma_{0}\right)^{-1 / 2} D_{X s_{\sigma}+\delta} \omega D_{X s_{\sigma}+\delta}^{\dagger / 2} \mathcal{N}_{0}\left(\sigma_{0}\right)^{-1 / 2}\right] \\
= & D_{s_{\sigma}}^{\dagger} \mathcal{P}_{\sigma_{0}, \mathcal{N}_{0}}\left(D_{X s_{\sigma}+\delta} \omega D_{X s_{\sigma}+\delta}^{\dagger}\right) D_{s_{\sigma}} .
\end{aligned}
$$

For the first equality, we use the definition of the Petz map and Ansatz 1. The second equality follows from (31)-(34) and the fact that $f\left(U A U^{\dagger}\right)=U f(A) U^{\dagger}$ for a function $f$, a unitary operator $U$, and a Hermitian operator $A$. The third equality follows because $D_{\delta} D_{X s_{\sigma}+\delta}^{\dagger}=D_{X s_{\sigma}}^{\dagger} e^{i \phi}$ for $\phi$ a phase. The fourth equality follows from the adjoint channel covariance relation in (36) and Ansatz 3. The fifth equality follows because $D_{s_{\sigma}} D_{X-1}^{\dagger}\left(X s_{\sigma}\right)=$ $e^{i \varphi} I$ for some phase $\varphi$. The final equality follows by recognizing the form of the Petz map $\mathcal{P}_{\sigma_{0}, \mathcal{N}_{0}}$, corresponding to the zero-mean state $\sigma_{0}$ and the zero-displacement channel $\mathcal{N}_{0}$.

The above reasoning suggests that we should focus on determining an explicit form for $\mathcal{P}_{\sigma_{0}, \mathcal{N}_{0}}(\omega)$. That is, the above reasoning suggests that an arbitrary Petz map $\mathcal{P}_{\sigma, \mathcal{N}}$ can be realized as a serial concatenation of the displacement $D_{X s_{\sigma}+\delta}$, the Petz map $\mathcal{P}_{\sigma_{0}, \mathcal{N}_{0}}$, and the displacement $D_{s_{\sigma}}^{\dagger}$. After we give an explicit form for $\mathcal{P}_{\sigma_{0}, \mathcal{N}_{0}}$ as a quantum Gaussian channel with matrices $X_{P}$ and $Y_{P}$, it should become clear why the displacement $\delta_{P}$ in the Petz map $\mathcal{P}_{\sigma, \mathcal{N}}$ has the form in (46).

\subsection{Step 2: Deducing a hypothesis for an explicit form for the Petz map, by considering Gaussian input states}

In this step, we continue working with Ansatzes 1-3, with our main objective being to arrive at a hypothesis for the action of the Petz recovery map $\mathcal{P}_{\sigma_{0}, \mathcal{N}_{0}}$ on the mean vector and covariance matrix of an input Gaussian state. Here we consider the serial concatenation of the three completely positive maps in (53)-(55). We begin by considering the action of the last completely positive map on a zero-mean Gaussian input state $\omega_{0}$. To this end, recall from [5, Appendix C] that if $\omega_{0}$ and $\sigma_{0}$ are zero-mean Gaussian states, then $\sqrt{\sigma_{0}} \omega_{0} \sqrt{\sigma_{0}}$ is an (unnormalized) Gaussian operator with zero mean vector and covariance matrix given by

$$
V_{\sqrt{\sigma_{0}} \omega_{0} \sqrt{\sigma_{0}}}=V_{\sigma_{0}}-\left(V_{\sqrt{\sigma_{0}}}-V_{\sigma_{0}}\right)\left(V_{\omega_{0}}+V_{\sigma_{0}}\right)^{-1}\left(V_{\sqrt{\sigma_{0}}}-V_{\sigma_{0}}\right) .
$$

Applying a formula from [5, Appendix B-2] (while noting our different convention for Gaussian states), we find that

$$
V_{\sqrt{\sigma_{0}}}=\left(\sqrt{I+\left(V_{\sigma_{0}} \Omega\right)^{-2}}+I\right) V_{\sigma_{0}}
$$


which is a symmetric matrix because $V_{\sigma_{0}}$ is. Indeed, consider that

$$
\begin{aligned}
V_{\sqrt{\sigma_{0}}}^{T} & =\left[\left(\sqrt{I+\left(V_{\sigma_{0}} \Omega\right)^{-2}}+I\right) V_{\sigma_{0}}\right]^{T}=V_{\sigma_{0}}\left(\sqrt{I+\left(\Omega V_{\sigma_{0}}\right)^{-2}}+I\right) \\
& =\Omega^{-1} \Omega V_{\sigma_{0}}\left(\sqrt{I+\left(\Omega V_{\sigma_{0}}\right)^{-2}}+I\right)=\Omega^{-1}\left(\sqrt{I+\left(\Omega V_{\sigma_{0}}\right)^{-2}}+I\right) \Omega V_{\sigma_{0}} \\
& =\left(\sqrt{\Omega^{-1}\left[I+\left(\Omega V_{\sigma_{0}}\right)^{-2}\right] \Omega}+I\right) V_{\sigma_{0}}=\left(\sqrt{\left[I+\left(\Omega^{-1} \Omega V_{\sigma_{0}} \Omega\right)^{-2}\right]}+I\right) V_{\sigma_{0}} \\
& =\left(\sqrt{I+\left(V_{\sigma_{0}} \Omega\right)^{-2}}+I\right) V_{\sigma_{0}}=V_{\sqrt{\sigma_{0}}} .
\end{aligned}
$$

The equality in (63) implies that

$$
V_{\sqrt{\sigma_{0}}}-V_{\sigma_{0}}=\sqrt{I+\left(V_{\sigma_{0}} \Omega\right)^{-2}} V_{\sigma_{0}}
$$

and in turn, after substituting into (62), that

$$
V_{\sqrt{\sigma_{0}} \omega_{0} \sqrt{\sigma_{0}}}=V_{\sigma_{0}}-\sqrt{I+\left(V_{\sigma_{0}} \Omega\right)^{-2}} V_{\sigma_{0}}\left(V_{\omega_{0}}+V_{\sigma_{0}}\right)^{-1} V_{\sigma_{0}} \sqrt{I+\left(\Omega V_{\sigma_{0}}\right)^{-2}} .
$$

Thus, (69) establishes the action of the completely positive map $(\cdot) \rightarrow \sqrt{\sigma_{0}}(\cdot) \sqrt{\sigma_{0}}$ on an arbitrary zero-mean Gaussian state $\omega_{0}$.

From this discussion we already start seeing that the Petz map constructed out of a Gaussian state $\sigma$ and a Gaussian channel $\mathcal{N}$ should send normalized Gaussian states to normalized Gaussian states, because (i) conjugation by the square root of a Gaussian state (or the inverse square root of a Gaussian state as we will see) preserves the Gaussian form; (ii) the adjoint of a Gaussian channel is still Gaussian; and (iii) the Petz map is a priori known to be trace-preserving whenever $\mathcal{N}(\sigma)$ is a faithful state $[50,51,44]$. Then, [45, Theorem III.1] ensures that $\mathcal{P}$ must act as in $(26)$, for some $X_{P}, Y_{P}$, and $\delta_{P}$ to be determined.

With this preliminary identity in hand, we are ready to determine a hypothesis for the explicit action of $\mathcal{P}_{\sigma_{0}, \mathcal{N}_{0}}$. For the sake of simplicity, we consider the input Gaussian state to have vanishing first moments. In any case, since we are working to deduce a hypothesis for an explicit form for the Petz map, this is by no means a loss of generality. By applying (69) and Ansatz 2 (that the following density operator transformation $\omega \rightarrow \omega^{-1}$ induces the transformation $V_{\omega} \rightarrow-V_{\omega}$ on the level of covariance matrices), we can conclude that the completely positive map in (53) has the following effect on covariance matrices:

$$
\begin{aligned}
& V{\sqrt{\mathcal{N}_{0}\left(\sigma_{0}\right)}}^{-1} \omega_{0}{\sqrt{\mathcal{N}_{0}\left(\sigma_{0}\right)}}^{-1} \\
& =-V_{\mathcal{N}(\sigma)}-\sqrt{I+\left(V_{\mathcal{N}(\sigma)} \Omega\right)^{-2}} V_{\mathcal{N}(\sigma)}\left(V_{\omega}-V_{\mathcal{N}(\sigma)}\right)^{-1} V_{\mathcal{N}(\sigma)} \sqrt{I+\left(\Omega V_{\mathcal{N}(\sigma)}\right)^{-2}} .
\end{aligned}
$$

In the above, we have also used the identities $V_{\mathcal{N}_{0}\left(\sigma_{0}\right)}=V_{\mathcal{N}(\sigma)}$ and $V_{\omega_{0}}=V_{\omega}$. So now we consider further concatenating with the completely positive map in (54), by applying (30) and Ansatz 3 (that $X$ is invertible):

$$
\begin{aligned}
& \left.V_{\mathcal{N}_{0}^{\dagger}\left(\sqrt{\mathcal{N}_{0}\left(\sigma_{0}\right)}-1\right.} \omega_{0} \sqrt{\mathcal{N}_{0}\left(\sigma_{0}\right)}-1\right)= \\
& \quad X^{-1}\left[-V_{\mathcal{N}(\sigma)}-\sqrt{I+\left(V_{\mathcal{N}(\sigma)} \Omega\right)^{-2}} V_{\mathcal{N}(\sigma)}\left(V_{\omega}-V_{\mathcal{N}(\sigma)}\right)^{-1} V_{\mathcal{N}(\sigma)} \sqrt{I+\left(\Omega V_{\mathcal{N}(\sigma)}\right)^{-2}}+Y\right] X^{-T} .
\end{aligned}
$$


But consider that $V_{\mathcal{N}(\sigma)}=X V_{\sigma} X^{T}+Y$, so that (71) simplifies as follows:

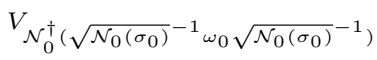

$$
\begin{aligned}
& =X^{-1}\left[-\left(X V_{\sigma} X^{T}+Y\right)\right. \\
& \left.-\sqrt{I+\left(V_{\mathcal{N}(\sigma)} \Omega\right)^{-2}} V_{\mathcal{N}(\sigma)}\left(V_{\omega}-V_{\mathcal{N}(\sigma)}\right)^{-1} V_{\mathcal{N}(\sigma)} \sqrt{I+\left(\Omega V_{\mathcal{N}(\sigma)}\right)^{-2}}+Y\right] X^{-T} \\
& =X^{-1}\left[-X V_{\sigma} X^{T}-\sqrt{I+\left(V_{\mathcal{N}(\sigma)} \Omega\right)^{-2}} V_{\mathcal{N}(\sigma)}\left(V_{\omega}-V_{\mathcal{N}(\sigma)}\right)^{-1} V_{\mathcal{N}(\sigma)} \sqrt{I+\left(\Omega V_{\mathcal{N}(\sigma)}\right)^{-2}}\right] X^{-T} \\
& =-V_{\sigma}-X^{-1} \sqrt{I+\left(V_{\mathcal{N}(\sigma)} \Omega\right)^{-2}} V_{\mathcal{N}(\sigma)}\left(V_{\omega}-V_{\mathcal{N}(\sigma)}\right)^{-1} V_{\mathcal{N}(\sigma)} \sqrt{I+\left(\Omega V_{\mathcal{N}(\sigma)}\right)^{-2}} X^{-T} .
\end{aligned}
$$

So then we can finally consider the serial concatenation of the three completely positive maps in (53)-(55):

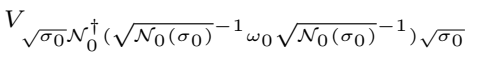

$$
\begin{aligned}
& =V_{\sigma}-\sqrt{I+\left(V_{\sigma} \Omega\right)^{-2}} V_{\sigma} \\
& \times\left(-V_{\sigma}-X^{-1} \sqrt{I+\left(V_{\mathcal{N}(\sigma)} \Omega\right)^{-2}} V_{\mathcal{N}(\sigma)}\left(V_{\omega}-V_{\mathcal{N}(\sigma)}\right)^{-1} V_{\mathcal{N}(\sigma)} \sqrt{I+\left(\Omega V_{\mathcal{N}(\sigma)}\right)^{-2}} X^{-T}+V_{\sigma}\right)^{-1} \\
& \times V_{\sigma} \sqrt{I+\left(\Omega V_{\sigma}\right)^{-2}} \\
& =V_{\sigma}-\sqrt{I+\left(V_{\sigma} \Omega\right)^{-2}} V_{\sigma} \\
& \times\left(-X^{-1} \sqrt{I+\left(V_{\mathcal{N}(\sigma)} \Omega\right)^{-2}} V_{\mathcal{N}(\sigma)}\left(V_{\omega}-V_{\mathcal{N}(\sigma)}\right)^{-1} V_{\mathcal{N}(\sigma)} \sqrt{I+\left(\Omega V_{\mathcal{N}(\sigma)}\right)^{-2}} X^{-T}\right)^{-1} \\
& \times V_{\sigma} \sqrt{I+\left(\Omega V_{\sigma}\right)^{-2}}
\end{aligned}
$$

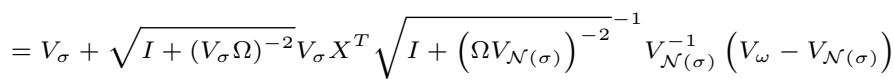

$$
\begin{aligned}
& \times V_{\mathcal{N}(\sigma)}^{-1}{\sqrt{I+\left(V_{\mathcal{N}(\sigma)} \Omega\right)^{-2}}}^{-1} X V_{\sigma} \sqrt{I+\left(V_{\sigma} \Omega\right)^{-2}} .
\end{aligned}
$$

An inspection of (76) above suggests that the Petz map $\mathcal{P}_{\sigma_{0}, \mathcal{N}_{0}}$ is a quantum Gaussian channel with the following action on an input covariance matrix $V_{\omega}$ :

$$
V_{\mathcal{P}_{\sigma_{0}, \mathcal{N}_{0}}\left(\omega_{0}\right)}=X_{P} V_{\omega} X_{P}^{T}+Y_{P}
$$

where

$$
\begin{aligned}
X_{P} & \equiv \sqrt{I+\left(V_{\sigma} \Omega\right)^{-2}} V_{\sigma} X^{T} \sqrt{I+\left(\Omega V_{\mathcal{N}(\sigma)}\right)^{-2}} V_{\mathcal{N}(\sigma)}^{-1}, \\
Y_{P} & \equiv V_{\sigma}-X_{P} V_{\mathcal{N}(\sigma)} X_{P}^{T} .
\end{aligned}
$$

Combining with the development in Section 3.1, the results in (77), (61) and [45, Theorem III.1] imply that in general

$$
\mathcal{P}_{\sigma, \mathcal{N}}:\left\{\begin{array}{rll}
V & \longmapsto X_{P} V X_{P}^{T}+Y_{P} \\
s & \longmapsto X_{P} s+\delta_{P}
\end{array},\right.
$$

where

$$
\delta_{P} \equiv s_{\sigma}-X_{P}\left(X s_{\sigma}+\delta\right)
$$


and $\delta$ is the vector appearing in (26); it follows because

$$
\mathcal{P}_{\sigma, \mathcal{N}}(\omega)=D_{s_{\sigma}}^{\dagger} \mathcal{P}_{\sigma_{0}, \mathcal{N}_{0}}\left(D_{X s_{\sigma}+\delta} \omega D_{X s_{\sigma}+\delta}^{\dagger}\right) D_{s_{\sigma}},
$$

which implies that

$$
s_{\mathcal{P}_{\sigma, \mathcal{N}}(\omega)}=X_{P}\left(s_{\omega}-X s_{\sigma}-\delta\right)+s_{\sigma} .
$$

So by using Ansatzes 1-3, we have arrived at our hypothesis (80) for the Gaussian form of the Petz map $\mathcal{P}_{\sigma, \mathcal{N}}$. In [30], we give the final steps of the proof that the Gaussian channel specified in (80) is indeed equal to the Petz map $\mathcal{P}_{\sigma, \mathcal{N}}$.

\section{Conclusion}

The main result of this paper is Theorem 1, which establishes an explicit form for the Petz map as a bosonic Gaussian channel whenever the state $\sigma$ and the channel $\mathcal{N}$ are bosonic Gaussian. Our proof approach is first to consider three ansatzes in order to arrive at a hypothesis for the Gaussian form of the Petz map. These ansatzes included 1) working with the form of the Petz map in (8) in spite of the fact that $[\mathcal{N}(\sigma)]^{-1}$ is an unbounded operator, 2) negating the covariance matrix of the Gaussian state $\sigma$ if $\sigma$ is inverted, and 3) assuming that the $X$ matrix in (25), corresponding to a Gaussian channel, is invertible. After deducing a hypothesis for an explicit form, [30] proves that this hypothesis is in fact correct, by demonstrating that the Gaussian Petz channel satisfies the equations in [30, Equation 3.107] for all bounded operators $A$ and $B$.

In future work, it would be interesting to determine whether the following inequality, considered in $[7,59]$, could be satisfied whenever all of the objects involved are Gaussian:

$$
D(\rho \| \sigma) \geq D(\mathcal{N}(\rho) \| \mathcal{N}(\sigma))-\log F\left(\rho,\left(\mathcal{P}_{\sigma, \mathcal{N}} \circ \mathcal{N}\right)(\rho)\right) .
$$

More generally, one could consider the various inequalities proposed in [8] for the Gaussian case.

Acknowledgements. We thank Gerardo Adesso, Prabha Mandayam, Alessio Serafini, Kaushik Seshadreesan, and Andreas Winter for discussions related to this paper. LL thanks Davide Orsucci for his contribution to the proof contained in [30, Section 3.4].

\section{References}

1 Gerardo Adesso, Sammy Ragy, and Antony R. Lee. Continuous variable quantum information: Gaussian states and beyond. Open Systems and Information Dynamics, 21(0102):1440001, June 2014. arXiv:1401.4679.

2 Alvaro M. Alhambra, Stephanie Wehner, Mark M. Wilde, and Mischa P. Woods. Work and reversibility in quantum thermodynamics, June 2015. arXiv:1506.08145.

3 Alvaro M. Alhambra and Mischa P. Woods. Dynamical maps, quantum detailed balance and Petz recovery map, September 2016. arXiv:1609.07496.

4 Hans-A. Bachor and Timothy C. Ralph. A Guide to Experiments in Quantum Optics. Wiley, second edition, March 2004.

5 Leonardo Banchi, Samuel L. Braunstein, and Stefano Pirandola. Quantum fidelity for arbitrary Gaussian states. Physical Review Letters, 115(26):260501, December 2015. arXiv:1507.01941. doi:10.1103/PhysRevLett.115.260501. 
6 J. Bergh and Jorgen Löfström. Interpolation Spaces. Springer-Verlag Berlin Heidelberg, 1976.

7 Mario Berta, Kaushik Seshadreesan, and Mark M. Wilde. Rényi generalizations of the conditional quantum mutual information. Journal of Mathematical Physics, 56(2):022205, February 2015. arXiv:1403.6102.

8 Mario Berta, Kaushik P. Seshadreesan, and Mark M. Wilde. Rényi generalizations of quantum information measures. Physical Review A, 91(2):022333, February 2015. arXiv:1502.07977. doi:10.1103/PhysRevA.91.022333.

9 Fernando G. S. L. Brandao, Matthias Christandl, and Jon Yard. Faithful squashed entanglement. Communications in Mathematical Physics, 306(3):805-830, September 2011. arXiv:1010.1750. doi:10.1007/s00220-011-1302-1.

10 Fernando G. S. L. Brandao, Aram W. Harrow, Jonathan Oppenheim, and Sergii Strelchuk. Quantum conditional mutual information, reconstructed states, and state redistribution. Physical Review Letters, 115(5):050501, July 2014. arXiv:1411.4921.

11 Francesco Buscemi, Siddhartha Das, and Mark M. Wilde. Approximate reversibility in the context of entropy gain, information gain, and complete positivity. Physical Review A, 93(6):062314, June 2016. arXiv:1601.01207. doi:10.1103/PhysRevA .93.062314.

12 Filippo Caruso, Jens Eisert, Vittorio Giovannetti, and Alexander S. Holevo. Multi-mode bosonic Gaussian channels. New Journal of Physics, 10:083030, August 2008. arXiv:0804.0511.

13 Xiao-yu Chen. Gaussian relative entropy of entanglement. Physical Review A, 71(6):062320, June 2005. arXiv:quant-ph/0402109. doi:10.1103/PhysRevA.71.062320.

14 Matthias Christandl and Andreas Winter. "Squashed entanglement" - an additive entanglement measure. Journal of Mathematical Physics, 45(3):829-840, March 2004. arXiv:quant$\mathrm{ph} / 0308088$.

15 Igor Devetak and Jon Yard. Exact cost of redistributing multipartite quantum states. Physical Review Letters, 100(23):230501, June 2008. doi:10.1103/PhysRevLett.100. 230501.

16 Frederic Dupuis and Mark M. Wilde. Swiveled Rényi entropies. Quantum Information Processing, 15(3):1309-1345, March 2016. arXiv:1506.00981.

17 Edward G. Effros. A matrix convexity approach to some celebrated quantum inequalities. Proceedings of the National Academy of Sciences of the United States of America, 106(4):1006-1008, January 2009. arXiv:0802.1234.

18 Omar Fawzi and Renato Renner. Quantum conditional mutual information and approximate Markov chains. Communications in Mathematical Physics, 340(2):575-611, December 2015. arXiv:1410.0664.

19 Marco G. Genoni, Ludovico Lami, and Alessio Serafini. Conditional and unconditional Gaussian quantum dynamics. Contemporary Physics, 57(3):331-349, January 2016. arXiv:1607.02619. doi:10.1080/00107514.2015.1125624.

20 Christopher Gerry and Peter Knight. Introductory Quantum Optics. Cambridge University Press, November 2004.

21 Vittorio Giovannetti, Raul Garcia-Patron, Nicolas J. Cerf, and Alexander S. Holevo. Ultimate classical communication rates of quantum optical channels. Nature Photonics, 8:796800, September 2014. arXiv:1312.6225.

22 Vittorio Giovannetti, Saikat Guha, Seth Lloyd, Lorenzo Maccone, Jeffrey H. Shapiro, and Horace P. Yuen. Classical capacity of the lossy bosonic channel: The exact solution. Physical Review Letters, 92(2):027902, January 2004. arXiv:quant-ph/0308012. doi:10.1103/PhysRevLett.92.027902.

23 Vittorio Giovannetti, Alexander S. Holevo, and Raul Garcia-Patron. A solution of Gaussian optimizer conjecture for quantum channels. Communications in Mathematical Physics, 334(3):1553-1571, March 2015. arXiv:1312.2251. 
24 Patrick Hayden, Richard Jozsa, Denes Petz, and Andreas Winter. Structure of states which satisfy strong subadditivity of quantum entropy with equality. Communications in Mathematical Physics, 246(2):359-374, April 2003. arXiv:quant-ph/0304007.

25 Isidore Isaac Hirschman. A convexity theorem for certain groups of transformations. Journal d'Analyse Mathématique, 2(2):209-218, December 1952.

26 Oscar Lanford III and Derek W. Robinson. Mean entropy of states in quantum-statistical mechanics. Journal of Mathematical Physics, 9(7):1120-1125, July 1968.

27 Marius Junge, Renato Renner, David Sutter, Mark M. Wilde, and Andreas Winter. Universal recovery from a decrease of quantum relative entropy, September 2015. arXiv:1509.07127.

28 Isaac H. Kim. Operator extension of strong subadditivity of entropy. Journal of Mathematical Physics, 53(12):122204, December 2012. arXiv:1210.5190.

29 Isaac H. Kim. Application of conditional independence to gapped quantum many-body systems, 2013. http://www.physics.usyd.edu.au/quantum/Coogee2013.

30 Ludovico Lami, Siddhartha Das, and Mark M. Wilde. Approximate reversal of quantum Gaussian dynamics, 2017. arXiv:1702.04737.

31 Matthew S. Leifer and Robert W. Spekkens. Towards a formulation of quantum theory as a causally neutral theory of Bayesian inference. Physical Review A, 88(5):052130, November 2013. arXiv:1107.5849. doi:10.1103/PhysRevA.88.052130.

32 Marius Lemm and Mark M. Wilde. Information-theoretic limitations on approximate quantum cloning and broadcasting, August 2016. arXiv:1608.07569.

$33 \mathrm{Ke} \mathrm{Li}$ and Andreas Winter. Squashed entanglement, $k$-extendibility, quantum Markov chains, and recovery maps, October 2014. arXiv:1410.4184.

34 Elliott H. Lieb. Convex trace functions and the Wigner-Yanase-Dyson conjecture. Advances in Mathematics, 11(3):267-288, December 1973.

35 Elliott H. Lieb and Mary Beth Ruskai. A fundamental property of quantum-mechanical entropy. Physical Review Letters, 30(10):434-436, March 1973.

36 Elliott H. Lieb and Mary Beth Ruskai. Proof of the strong subadditivity of quantum mechanical entropy. Journal of Mathematical Physics, 14(12):1938-1941, December 1973.

37 Göran Lindblad. Expectations and entropy inequalities for finite quantum systems. Communications in Mathematical Physics, 39(2):111-119, June 1974.

38 Göran Lindblad. Completely positive maps and entropy inequalities. Communications in Mathematical Physics, 40(2):147-151, June 1975. doi:10.1007/bf01609396.

39 Iman Marvian and Seth Lloyd. From clocks to cloners: Catalytic transformations under covariant operations and recoverability, August 2016. arXiv:1608.07325.

40 William Matthews, Stephanie Wehner, and Andreas Winter. Distinguishability of quantum states under restricted families of measurements with an application to quantum data hiding. Communications in Mathematical Physics, 291(3):813-843, November 2009. arXiv:0810.2327.

41 Milán Mosonyi. Entropy, Information and Structure of Composite Quantum States. $\quad \mathrm{PhD}$ thesis, Katholieke Universiteit Leuven, 2005. Available at https://lirias.kuleuven.be/bitstream/1979/41/2/thesisbook9.pdf.

42 Milán Mosonyi and Dénes Petz. Structure of sufficient quantum coarse-grainings. Letters in Mathematical Physics, 68(1):19-30, April 2004. arXiv:quant-ph/0312221. doi:10.1007/ s11005-004-4072-2.

43 Michael A. Nielsen and Denés Petz. A simple proof of the strong subadditivity inequality. Quantum Information and Computation, 5(6):507-513, September 2005. arXiv:quantph/0408130.

44 Masanori Ohya and Denes Petz. Quantum Entropy and Its Use. Springer-Verlag, 1993. 
45 Giacomo De Palma, Andrea Mari, Vittorio Giovannetti, and Alexander S. Holevo. Normal form decomposition for Gaussian-to-Gaussian superoperators. Journal of Mathematical Physics, 56(5):052202, May 2015. arXiv:1502.01870.

46 Giacomo De Palma, Dario Trevisan, and Vittorio Giovannetti. Gaussian states minimize the output entropy of one-mode quantum Gaussian channels, October 2016. arXiv:1610.09970.

47 Giacomo De Palma, Dario Trevisan, and Vittorio Giovannetti. One-mode quantum-limited Gaussian channels have Gaussian maximizers, October 2016. arXiv:1610.09967.

48 Giacomo De Palma, Dario Trevisan, and Vittorio Giovannetti. Gaussian states minimize the output entropy of the one-mode quantum attenuator. IEEE Transactions on Information Theory, 63(1):728-737, January 2017. arXiv:1605.00441.

49 Gh.-S. Paraoanu and Horia Scutaru. Fidelity for multimode thermal squeezed states. Physical Review A, 61(2):022306, January 2000. arXiv:quant-ph/9907068. doi:10.1103/ PhysRevA.61.022306.

50 Denes Petz. Sufficient subalgebras and the relative entropy of states of a von Neumann algebra. Communications in Mathematical Physics, 105(1):123-131, 1986.

51 Denes Petz. Sufficiency of channels over von Neumann algebras. Quarterly Journal of Mathematics, 39(1):97-108, 1988.

52 Denes Petz. Monotonicity of quantum relative entropy revisited. Reviews in Mathematical Physics, 15(01):79-91, March 2003. arXiv:quant-ph/0209053.

53 Stefano Pirandola, Riccardo Laurenza, Carlo Ottaviani, and Leonardo Banchi. Fundamental limits of repeaterless quantum communications, September 2016 . arXiv:1510.08863v6.

54 Haoyu Qi and Mark M. Wilde. Capacities of quantum amplifier channels. Physical Review A, 95(1):012339, January 2017. arXiv:1605.04922.

55 Derek W. Robinson and David Ruelle. Mean entropy of states in classical statistical mechanics. Communications in Mathematical Physics, 5(4):288-300, August 1967.

56 Mary Beth Ruskai. Inequalities for quantum entropy: a review with conditions for equality. Journal of Mathematical Physics, 43:4358-4375, 2002. erratum 46, 019901 (2005); arXiv:quant-ph/0205064.

57 Stefan Scheel and Dirk-Gunnar Welsch. Entanglement generation and degradation by passive optical devices. Physical Review A, 64(6):063811, November 2001. arXiv:quantph/0103167. doi:10.1103/PhysRevA.64.063811.

58 Alessio Serafini. Quantum Continuous Variables. CRC Press, 2017.

59 Kaushik P. Seshadreesan, Mario Berta, and Mark M. Wilde. Rényi squashed entanglement, discord, and relative entropy differences. Journal of Physics A: Mathematical and Theoretical, 48(39):395303, September 2015. arXiv:1410.1443.

60 R. Simon, N. Mukunda, and Biswadeb Dutta. Quantum-noise matrix for multimode systems: $U(n)$ invariance, squeezing, and normal forms. Physical Review A, 49(3):1567-1583, March 1994. doi:10.1103/PhysRevA.49.1567.

61 David Sutter, Mario Berta, and Marco Tomamichel. Multivariate trace inequalities. Communications in Mathematical Physics, 352(1):37-58, May 2017. arXiv:1604.03023.

62 David Sutter, Omar Fawzi, and Renato Renner. Universal recovery map for approximate Markov chains. Proceedings of the Royal Society A, 472(2186), February 2016. arXiv:1504.07251.

63 David Sutter, Marco Tomamichel, and Aram W. Harrow. Strengthened monotonicity of relative entropy via pinched Petz recovery map. IEEE Transactions on Information Theory, 62(5):2907-2913, May 2016. arXiv:1507.00303.

64 Robert R. Tucci. Quantum entanglement and conditional information transmission, September 1999. arXiv:quant-ph/9909041. 
65 Robert R. Tucci. Entanglement of distillation and conditional mutual information, February 2002. arXiv:quant-ph/0202144.

66 Armin Uhlmann. Endlich dimensionale dichtmatrizen, ii. Wiss. Z. Karl-Marx-University Leipzig, 22(Jg. H. 2.):139, 1973.

67 Armin Uhlmann. The "transition probability" in the state space of a *-algebra. Reports on Mathematical Physics, 9(2):273-279, 1976.

68 Hisaharu Umegaki. Conditional expectations in an operator algebra IV (entropy and information). Kodai Mathematical Seminar Reports, 14(2):59-85, 1962.

69 Xiang-Bin Wang, Tohya Hiroshima, Akihisa Tomita, and Masahito Hayashi. Quantum information with Gaussian states. Physics Reports, 448(1-4):1-111, August 2007. arXiv:0801.4604. doi:10.1016/j.physrep.2007.04.005.

70 Xiang-Bin Wang, L. C. Kwek, and C. H. Oh. Bures fidelity for diagonalizable quadratic Hamiltonians in multi-mode systems. Journal of Physics A: Mathematical and General, 33(27):4925, July 2000. URL: http://stacks.iop.org/0305-4470/33/i=27/a=310.

71 Mark M. Wilde. Recoverability in quantum information theory. Proceedings of the Royal Society A, 471(2182):20150338, October 2015. arXiv:1505.04661.

72 Mark M. Wilde. Monotonicity of $p$-norms of multiple operators via unitary swivels, October 2016. arXiv:1610.01262.

73 Mark M. Wilde, Patrick Hayden, and Saikat Guha. Information trade-offs for optical quantum communication. Physical Review Letters, 108(14):140501, April 2012. arXiv:1105.0119.

74 Mark M. Wilde and Haoyu Qi. Energy-constrained private and quantum capacities of quantum channels, September 2016. arXiv:1609.01997.

75 Andreas Winter and Ke Li. A stronger subadditivity relation? with applications to squashed entanglement, sharability and separability. notes available online at http://www.maths.bris.ac.uk/ csajw/stronger_subadditivity.pdf , see also http://www.scribd.com/document/337859204, 2012.

76 Jon Yard and Igor Devetak. Optimal quantum source coding with quantum side information at the encoder and decoder. IEEE Transactions on Information Theory, 55(11):5339-5351, November 2009. arXiv:0706.2907. 